\title{
Is My Femininity a Liability? Longitudinal Associations between Girls' Experiences of Gender Discrimination, Internalizing Symptoms, and Gender Identity
}

\author{
Adam A. Rogers $\mathbb{B}^{1} \cdot$ Rachel E. Cook ${ }^{2} \cdot$ Kaitlyn Guerrero ${ }^{1}$ \\ Received: 27 October 2021 / Accepted: 1 January 2022 / Published online: 18 January 2022 \\ (c) The Author(s), under exclusive licence to Springer Science+Business Media, LLC, part of Springer Nature 2022
}

\begin{abstract}
Gender discrimination is a common experience for adolescent girls and has implications for their mental health and identity development. Guided by Phenomenological Variant of Ecological Systems Theory (PVEST; Spencer et al. 1997), this study examined the longitudinal and bidirectional associations between adolescent girls' experiences of gender discrimination, their internalizing symptoms, and gender identity. The sample was 161 adolescent girls (ages $\left.14-17 ; M_{\text {age }}=15.90\right)$ from across the United States (51\% White; 17\% African American, 11\% Hispanic/Latina) who participated in a short-term longitudinal study of adolescent development. The results showed a reciprocal, longitudinal association between discrimination and internalizing symptoms. Discrimination also predicted longitudinal declines in gender identity, which was explained indirectly through internalizing symptoms. The findings implicate gender discrimination as a distinct risk factor during girls' developmental years, and underscore the importance of helping girls learn adaptive responses to sexism, while also reducing actual occurrence and exposure.
\end{abstract}

Keywords Sexism $\cdot$ Discrimination $\cdot$ Adolescent $\cdot$ Mental Health $\cdot$ Gender Identity

\section{Introduction}

Gender discrimination refers to negative treatment of an individual on the basis of their gender expression or gender identity. It can include a range of experiences such as sexual harassment, stereotyping, and biased treatment from others. Most adolescent girls report experiencing gender-based discrimination, and these experiences span across their most salient social ecologies, such as peer, familial, academic, and athletic domains (Leaper \& Brown 2008). There is growing literature on girls' experiences with gender discrimination, though there is still an incomplete understanding of how girls' subjective experiences with gender discrimination impact two core features of their development: their emotional well-being

Adam A. Rogers

adam_rogers@byu.edu

1 School of Family Life, Brigham Young University, Provo, UT 84602, USA

2 Institute for Child Development and Family Relations, California State University, San Bernardino, CA, USA and their emergent social identities. To address this gap, this study examined how adolescent girls' subjective experiences with gender discrimination were associated with indices of their mental health and their gender identity in short-term longitudinal study.

\section{Gender Discrimination, Meaning Making, and Development}

Phenomenological Variant of Ecological Systems Theory (PVEST; Spencer et al., 1997) is a useful framework for understanding how gender discrimination can shape adolescent girls' development. Building on prominent ecological theories (e.g., Bronfenbrenner et al., 2006), PVEST places emphasis on how individuals make meaning of their experiences within ecological systems, and particularly systems of power and oppression. Specifically, it holds that the subjective experience of oppression (e.g., discrimination) elicits feelings of social and psychological dissonance that are fundamentally distressing, and which demand a coping response from the individual. This coping response involves the reshaping of a person's social identity as they try to make sense of their 
relationship to the systems of power in which they are embedded (Spencer et al., 1997).

Gender discrimination is a key stressor in adolescent girls' lives, particularly given how common it is. Somewhere between $70-80 \%$ of girls report experiencing discrimination (Leaper \& Brown, 2008). These experiences typically derive from peers, but can also come from teachers, school staff, and parents (Leaper \& Brown, 2018). As such, experiences of discrimination are a near ubiquitous experience for girls, as they cut across their primary developmental contexts. According to PVEST, these experiences put girls at odds with their social environments, challenging psychological resources and creating the need for a coping response that can shape the development of their relevant social identity (i.e., gender identity). However, there is very little research to inform these suppositions. Guided by PVEST, this study examined how adolescent girls' experiences of discrimination were longitudinally associated with changes in their mental health and their gender identities.

Although boys, too, can experience gender discrimination, this study focused on girls because of differences in the prevalence, subjective experience, and sociocultural implications of their experiences compared to boys'. As youth cope with gender discrimination, they respond not to categories or labels, but to their positioning within social systems. In most adolescent contexts in the U.S., there is greater inherent valuing of masculinity compared to femininity (Rogers et al., 2021c). Thus, compared to girls, boys' vulnerability to gender discrimination would likely be lower and their coping responses would differ.

\section{Gender Discrimination and Mental Health}

Gender discrimination may have implications for girls' mental health and psychological well-being. Research on discrimination more broadly (e.g., racial/ethnic discrimination) has documented robust links between subjective discriminatory experiences and internalizing challenges among youth. This is because discrimination can elicit social and psychological dissonance that challenges a youth's sense of self and places them at odds with their external environments. For example, individuals who experience discrimination can develop a reduced self-concept (Cassidy et al., 2004), a diminished sense of control within their environments, and weakened beliefs in justice and fairness (Kim \& Park, 2018). It can also reinforce a marginalized social position (DeLay et al., 2018) and the attenuation of interpersonal ties, especially among peers (Andrews et al., 2018; Baysu et al., 2014). The result is that discrimination can tie up valuable psychological resources and precipitate psychological distress, such as internalizing symptoms. Indeed, studies on racial/ethnic minority youth show robust links between their experiences of racial/ethnic discrimination and greater internalizing problems, such as depression, anxiety, and stress (Benner, 2017; Grollman, 2012).

Of course, caution is warranted when drawing parallels between gender discrimination and other forms of discrimination (e.g., racial/ethnic discrimination), given that there are unique social systems giving rise to these distinct forms of oppression (e.g., historical systems of white supremacy vs. patriarchal systems). However, research on diverse adult women has linked their experiences of gender discrimination with elevated depression and psychological distress, poorer mental functioning, and lower life satisfaction (Andersson \& Harnois, 2020). Currently, there is considerably less work on adolescent girls' appraisals of gender discrimination and the effects on their psychological functioning or mental health. This is a surprising omission given that adolescence ushers in cognitive and psychological advancements that render discrimination more discernable and more personally relevant. Girls are now more knowledgeable of gender-related concepts than when they were children, such as gender stereotypes (McKown \& Weinstein, 2003); and they have more complex moral reasoning, including about issues of equity (Killen et al., 2002). Given that adolescence is a sensitive period in which mental health difficulties are at their peak (Merikengas et al., 2010), it seems reasonable to expect that more regular experiences with gender discrimination are likely to precipitate emotional and psychological difficulties for girls.

Currently, most work on girls' experiences with gender discrimination has focused on other contexts, including academic contexts where it is explored in relation to girls' sense of belonging in school (e.g., Robnett, 2016; Rogers et al., 2021a), as well as their strategies for responding to sexism (Ayres et al., 2009; Leaper et al., 2012). However, even in these contexts, the implications for mental health are apparent. In a recent study on school-based gender discrimination by an adult (a teacher), girls in seventh grade reported higher levels of perceived school unfairness a year later, which was associated with more depressive symptoms (Bell \& Juvonen, 2020). The present study examined girls' broader experiences with discrimination (not confined to teachers or academic contexts). Specifically, it investigated the longitudinal and bidirectional associations between girls' experiences of discrimination and their internalizing symptoms, including symptoms of anxiety, depression, and loneliness.

\section{Gender Discrimination, Psychological Distress, and the Formation of a Gender Identity}

Processes of gender discrimination and the associated distress may also shape girls' gender identity development. 
Gender identity is a multi-dimensional construct representing an individual's subjective sense of belongingness to their gender in-group (Perry et al., 2019), such as the importance they place on their gender as a feature of their overall identity, their outward representation of their gender group, and their subjective experience of fit within that group. According to PVEST, the coping process stimulated by experiencing discrimination involves making meaning of one's sense of self in relation to the systems of oppression (i.e., patriarchal systems) in which they are embedded. Thus, to the extent that gender discrimination elicits internalizing symptoms, girls' coping efforts are likely to involve the shaping and reshaping of their gender identities. Simply put, if gender discrimination targets a characteristic that is less malleable ("I can't change my gender category"), it may lead to attempts to change one's social identity ("I can change my gender expression").

It is not entirely clear from the literature how these shifts in girls' identities might occur. In some instances, distressing experiences of discrimination could drive a greater identification with one's gender group. This could happen, for example, as girls seek members of their gender in-group as a source of social support, drawing empowerment and psychological resources for coping. Indeed, a strong sense of gender identity has been shown to be a key element of a feminist identity that proves protective in the face of discrimination (Leaper et al., 2012). These types of active coping responses-when enacted-have also been shown to be effective in buffering the negative psychological effects of discrimination (Ayres et al., 2009). Another possibility, although somewhat more speculative, is that experiences with oppression might lead youth to further internalize a socially marginalized identity. For example, a recent study found that gender nonconforming adolescents responded to peer harassment by further solidifying a less gender "typical" identity (DeLay et al., 2018). From this perspective, gender discrimination could lead girls to identify more with their gender in-group as they come to internalize sexist socialization.

However, there is also reason to expect that girls might instead distance themselves from their gender group in response to discrimination, and thus diminish in their sense of gender identity. Specifically, because discrimination targets a girl's gender category, girls who experience discrimination might come to perceive that identifying with femininity is a liability, especially in the context of patriarchal systems that devalue the feminine. To the extent that this is the case, adolescent girls might be motivated to psychologically and behaviorally dissociate from their gender group to avoid risk of future discrimination. There is evidence of this process among adult women in corporate spheres. In a large study examining women's experiences in male-dominated S.E.T career fields (Science, Engineering and Technology), the strong majority of women experienced gender discrimination in the workplace (Hewlett et al., 2008). A common coping strategy observed among these women was to enact more masculine-typed behavior and even dissociate from female colleagues, both in attempt to prevent future sexism (e.g., dressing more masculine to avoid sexual harassment) and to adapt to systems that devalued female contributions (e.g., a response to seeing few women in positions of leadership). Adolescent girls, too, navigate gender-biased institutions (e.g., schools) that take for granted patriarchal systems, and it is within these systems that girls must weigh the social costs of how they cope with sexism. Actively confronting discrimination could elicit backlash, escalate negative treatment, or incur dismissive responses from others (Kaiser \& Miller, 2004). In these contexts, girls may respond to distressing experiences of discrimination by reducing their identification with their gender collective rather than pay the social costs of active confrontation.

Ultimately, PVEST (Spencer et al., 1997) specifies that changes in identity can occur in response to psychological dissonance and distress. As such, this study also considered the possibility that these associations could be indirect in nature. That is, if shifts in gender identity represent a coping response to gender discrimination, one may reasonably expect their association to operate through internalizing symptoms. Therefore, in a follow-up step, the mediated effects of gender discrimination on gender identity through internalizing symptoms were examined.

Because gender identity is multi-dimensional, three indices of gender identity were examined in separate models. Gender centrality was assessed, which refers to a girl's perception of her gender as an important and salient feature of her overall personal identity. To the degree that an adolescent believes she is being discriminated against because of her identity as a girl, this may influence the importance gender in her overall sense of self. For example, she might downplay the importance of her gender in her overall identity to avoid any negative psychological implications of being part of a devalued group. Gender typicality was also examined, which indexes gender identity as one's subjective sense of similarity to girls, and their subjective sense of similarity to boys. It specifically assesses perceived similarity to gender groups across behavioral (time spent with friends, activities) and phenomenological (e.g., interests, feelings) components (Martin et al., 2017). One's selfperceived girl-typical and boy-typical expression may fluctuate in response to discrimination. If a girl's responds to discrimination by seeking support and solidarity with other girls, for example, she may come to perceive greater similarity to other girls over time. She may even perceive diminishing similarity to boys, who are the most common perpetrators of sexism (see Andrews et al., 2018). However, 
if feminine expression is perceived as a reason for discrimination in the first place, or as a risk for future discrimination, a girl may position herself as less feminine and/ or more masculine to adapt to this risk.

\section{Accounting for Background and Macro-Systemic Environments}

Gendered processes, and the subjective meaning of those experiences, can vary considerably for adolescents from different socio-demographic backgrounds. For example, gendered values and norms are often conveyed differently across cultural contexts, meaning that gender-based socialization can vary according to racial/ethnic backgrounds and identities (Crenshaw, 1991; Santos \& Toomey, 2018). Regarding discrimination in particular, negative treatment is often experienced on the basis of both ethnic and gender identities, which can precipitate unique disadvantages for ethnic minority girls (Grollman, 2012). However, race and ethnicity can also confound with social class, as white youth are disproportionately raised in economically advantaged environments (DeNavas-Walt et al., 2013). Finally, age may shape girls' appraisals of discrimination, which may be heightened with advancing cognitive development (Brown \& Bigler, 2005), as well as increasing gender-based pressures-such as sexualizing pressures-as girls mature physically (Leaper \& Brown, 2008). As a result, the analyses controlled for girls' ethnic identity, their mothers' highest level of formal education (an index of socioeconomic status), and their age.

A final consideration is the effect of the COVID-19 pandemic, which began shortly after the first wave of data collection. The pandemic has introduced a number of changes in adolescents' peer networks and family dynamics, such as school closures and even increased economic difficulties for their parents. These changes have altered interpersonal interactions and even created emotional difficulties for some (Rogers et al., 2021b). This study focused on interpersonal processes and emotional health, meaning that findings could be influenced by experiences with the pandemic. For these reasons, all analyses controlled for school format (e.g., remote vs. in-person learning) and reduced family income due to COVID-19.

\section{Current Study}

This study examined the longitudinal and bidirectional associations between girls' experiences of gender discrimination, their internalizing symptoms, and indices of their gender identity in a sample of girls aged 14-17. Specifically, it was guided by three goals and hypotheses. The first goal was to examine whether gender discrimination would longitudinally predict internalizing symptoms. Considerable data indicates that discrimination can undermine psychological and social resources for youth, but these suppositions have yet to be tested in relation to gender discrimination among girls. It was predicted that girls' experiences with discrimination would predict greater internalizing symptoms (depressive symptoms, anxiety symptoms, and loneliness) one year later, controlling for baseline levels (Hypothesis 1). The second goal was to determine if gender discrimination longitudinally predicted girls' sense of gender identity. PVEST (Spencer et al., 1997) asserts that the coping response to discrimination inherently involves the shaping and reshaping of one's relevant social identity. Therefore, it was predicted that gender discrimination would be associated with longitudinal changes in girls' sense of gender identity (Hypothesis 2). However, because studies are sparse, there was not enough data to support confident predictions about whether the broader trend would be toward greater or lesser identification with one's gender collective. As such, the predictions here remained exploratory. Finally, the last question was whether the association between gender discrimination and gender identity could be explained indirectly through internalizing symptoms. Changes in social identity are theorized to occur in response to the dissonance and distress elicited by discrimination (Spencer et al., 1997). Therefore, it was predicted that the longitudinal effects of gender discrimination on gender identity might operate through internalizing symptoms, representing a more indirect, or mediated process (Hypothesis 3).

\section{Method}

\section{Participants and Procedures}

Participants were 161 adolescent girls from across the United States, ranging in age from 14-17 $\left(M_{\text {age }}=15.90\right.$, $S D=1.26)$. They came from all regions of the U.S. (18\% Northeast; 22\% Midwest; 41\% South; 19\% West) and represented various racial/ethnic identities, including White $(50.9 \%, n=86)$, African American $(17.2 \%, n=$ 29), Hispanic/Latinx (11.2\%, $n=19)$, Asian American $(4.7 \%, n=8)$, and multiracial $(11.3 \%, n=19)$. Most identified as straight $(86 \%, n=141)$, though some identified as gay/lesbian $(4.3 \% ; n=7)$, bisexual $(8.5 \%, n=$ $12)$, or pansexual $(1.2 \%, n=1)$. They reported varying levels of mothers' formal education, including $25.0 \%$ with a high-school degree or less; $42.6 \%$ with at least some college, and $30.0 \%$ with a four-year college degree or higher. Just under half $(45.3 \%)$ reported receiving free or reduced meals at school. The participants were drawn from Project AHEAD (Advancing Health and Education 
in Adolescent Development), an ongoing longitudinal study of adolescent development. In October 2019, adolescents were recruited using a third-party research service, Bovitz ${ }^{\circledR}$, which retains a nationally representative panel of research participants. A stratified random sample of this panel was drawn using national quotas for gender, racial/ethnic identity, parent education, and geographic region. Inclusion criteria were that adolescents had to be between 14 and 17 years of age. Qualifying adolescents completed an initial baseline survey in October 2019, and then completed follow-up surveys every six months thereafter (April 2020, October 2020). At all waves, an email invitation was sent to all participants that included a link to the survey. Upon opening the invitation, parental consent and adolescent assent were obtained. Assenting adolescents were directed to the survey, which asked about their experiences and attitudes with academics, interpersonal relationships, and mental health. Surveys took approximately $30 \mathrm{~min}$ to complete, and adolescents were compensated with a $\$ 20$ Amazon e-gift card for participating. All procedures were approved by the Brigham Young University IRB.

Data for the present study were drawn from wave 1 (October '19) and wave 3 (October '20), representing a oneyear time lag. This was done because the measure of gender discrimination explicitly prompted participants to report experiences of discrimination in the past year, so as to allow adequate time for developmental change in these constructs to occur. For the present study, these timepoints are referred to as T1 (October '19) and T2 (October '20). Participants were only included if they identified as girls. Of 271 identifying girls who began the study in October 2019, 161 were retained by the third wave (or "Time 2"; 59\%) and comprised the analytic sample for this study. Attrition analyses (independent samples $t$-tests, chi square tests) showed no difference between those who attrited by wave 3 and those who were retained, both in terms of background characteristics (racial/ ethnic identity, age, mother education) and most key study variables (internalizing symptoms, gender identity indices). The exception is that those who attrited reported slightly higher levels of gender discrimination at Time $1(M=1.94$, $S D=0.82)$ than those who were retained $(M=1.66, S D=$ 0.81 , which amounted to a small effect size, $d=0.34$.

\section{Measures}

\section{Gender discrimination}

At both time-points (Oct 2019 and Oct 2020) girls' experiences of gender discrimination were measured using an adapted version of the Schedule of Sexist Events scale for use with adolescents (Leaper \& Brown, 2008). Participants indicated how often they had experienced six sexist events in the past year: receiving unwanted or inappropriate romantic attention; being told embarrassing or mean jokes about girls; being called a nasty or demeaning name related to being a girl; being teased about their physical appearance; receiving unwanted physical contact; and being teased or threatened with harm by a male. Each of these items were reported on their frequency of occurrence using a 4-point scale $(1=n o-$ never happened, $2=$ yes - happened once or twice, $3=$ yesa few times, $4=y e s-$ several times). These six items were averaged such that higher scores indicated more numerous experiences with gender discrimination $\left(\alpha_{\mathrm{t} 1}=0.89 ; \alpha_{\mathrm{t} 3}=\right.$ 0.93 ). The convergent and predictive validity of the original Schedule of Sexist Events Scale has been demonstrated previously (Klonoff \& Landrine, 1995), and the adolescent adaptation has been used reliably in developmental work (e.g., Leaper \& Brown, 2008).

\section{Depressive symptoms}

Participants reported their depressive symptoms using the Children's Depression Inventory-Short version (Allgaier et al. 2012). Participants completed 12 items indicating how often they had experienced symptoms in the past two weeks (e.g., "I feel cranky all the time" and "I am sad") on a rating scale of 1 (rarely or none of the time) to 4 (most of the time). Items were averaged such that higher scores indicated more severe depressive symptoms $\left(\alpha_{\mathrm{T} 1}=0.84, \alpha_{\mathrm{T} 2}=0.87\right)$.

\section{Anxiety symptoms}

Participants' anxiety symptoms were measured using the seven-item Generalized Anxiety Disorder Scale (GAD-7; Spitzer et al. 2006) which has been shown to produce valid scores among adolescent respondents (Tiirikainen et al. 2019). Participants rated their symptoms over the past seven days (e.g., "feeling nervous, anxious, or on edge") on a 4-point rating scale $(1=$ never, $4=$ nearly every day $)$. Items were averaged such that higher scores indicated higher levels of anxiety $\left(\alpha_{\mathrm{T} 1}=0.92, \alpha_{\mathrm{T} 2}=0.95\right)$.

\section{Loneliness}

Adolescents reported the degree of loneliness using the Three-Item Loneliness Scale (Hughes et al., 2004). Participants responded to three items (e.g., "I feel left out") on a rating scale of 1 (hardly ever) to 3 (often). Items were averaged such that higher scores indicated greater loneliness $\left(\alpha_{\mathrm{T} 3}=0.86, \alpha_{\mathrm{T} 2}=0.86\right)$.

\section{Gender identity centrality}

Adolescents reported how personally salient their gender was to their overall identity. Three items were drawn from 
the Multidimensional Inventory of Black Identity scale (Scottham et al. 2008), a measure of ethnic identity adapted to reflect identification with one's gender group. These items included, "I feel close to other girls", "I have a strong sense of belonging to other girls" and "If I were to describe myself to someone, one of the first ways I would describe myself is that I am a girl." Participants responded on a 5 -point rating scale $(1=$ strongly disagree, $5=$ strongly agree). Items were averaged so higher scores reflected greater centrality of gender to one's overall identity. Internal consistency was adequate for these items, $\alpha_{\mathrm{T} 1}=0.71 ; \alpha_{\mathrm{T} 2}$ $=0.78$.

\section{Similarity to girls and boys}

Participants completed the Perceived Similarity to Gender Groups scale developed by Martin and colleagues (2017). This scale consisted of two subscales: perceived similarity to girls and perceived similarity to boys. Each of these subscales consisted of five items assessing the degree of to which participants felt they typified girls (e.g., "how similar do you feel to girls?" and "how much do you act like girls?") or boys (e.g., "how similar do you feel to boys?" and "how much do you act like boys?"), respectively. Their responses on these five items, which were recorded on a 5-point rating scale $(1=$ not much, $5=a$ lot), were averaged such that higher scores reflected greater identification with each respective gender group. Internal consistency for similarity to girls $\left(\alpha_{\mathrm{T} 1}=0.92\right.$; $\left.\alpha_{\mathrm{T} 2}=0.89\right)$ and similarity to boys $\left(\alpha_{\mathrm{T} 1}=0.85 ; \alpha_{\mathrm{T} 2}=0.83\right)$ was adequate at both time points.

\section{Ethnic/racial identity}

Adolescents were instructed to mark all options that described their ethnic or racial identity from seven categories: Black or African American, Asian-American, Hispanic/Latino, White/Caucasian, American Indian or Alaska Native, Mixed/Biracial, or "other". Because of relatively small cell sizes, there was concern about the lack of statistical power when comparing adolescents from different ethnic groups. To avoid Type II error in the analyses, participant responses were recoded to reflect ethnic minority status $(0=$ non-Hispanic Caucasian; $1=$ non-White ethnic minority).

\section{Mothers' level of formal education}

Participants indicated their mothers' highest level of formal education on a 9-point scale: $(1=$ no formal schooling, $2=$ less than high school, $3=$ high School/GED, 4= some college, 5=2-year college degree, $6=4$-year college degree, $7=$ Master's degree, $8=$ Doctoral degree, $9=$ Professional degree, i.e., $J D, M D)$.
Age

Participants reported their age in years.

\section{School format}

Because Time 2 (October 2020) was administered in the midst of the COVID-19 pandemic, the majority of participants were receiving their schooling remotely/online $(70.1 \% ; n=115)$. Therefore, a control was included for school format $(0=$ in-person or hybrid, $1=$ fully remotel online).

\section{Reduced family income}

Similarly, a sizable minority $(45.6 \% ; n=77)$ reported that their families had experienced reduced income as a result of COVID-19. This was included as a covariate as well $(0=$ no reduced income, $1=$ yes, reduced income).

\section{Analytic Strategy}

Data were first screened for skewness, kurtosis, multivariate outliers, and missing data patterns to confirm that analytic assumptions were satisfied. Then, descriptive statistics (means and standard deviations) and zero-order correlations among key study variables were calculated to examine preliminary patterns in the data.

To examine our first and second hypotheses regarding the longitudinal, bidirectional associations between discrimination, internalizing symptoms and gender identity, a series of auto-regressive cross-lagged path models were estimated. This technique was used because it allowed for the simultaneous estimate of several regression equations, providing a more rigorous, multivariate test of the hypothesized associations to identify unique effects. Importantly, it also allowed for the estimation of prospective relations among the variables (e.g., discrimination predicting later internalizing distress; cross-lagged paths) while controlling for prior levels of those same constructs (auto-regressive paths), meaning that the cross-lagged paths are estimates of actual rank-order (between-person) change in the constructs of interest. Because the three subscales representing mental health difficulties were highly intercorrelated (depressive symptoms, anxiety symptoms, loneliness; $r$ s ranging from 0.64 to $0.69, p s<0.001$ ), they were averaged to form a single composite variable representing internalizing symptoms. This allowed for a more parsimonious approach to the data and a reduction in the overall number of models estimated. However, because the three indicators of gender identity (centrality, similarity to girls, similarity to boys) are more conceptually and empirically distinct, these were not used to create a composite variable, but instead remained as 
separate variables. Ultimately, three models were estimated, one for each indicator of gender identity.

Each path model included auto-regressive paths predicting each $\mathrm{T} 2$ variable by itself at $\mathrm{T} 1$. Then, six crosslagged paths were specified to examine the longitudinal, bidirectional effects of each construct on the others over time: $\mathrm{T} 1$ discrimination predicting $\mathrm{T} 2$ internalizing symptoms; $\mathrm{T} 1$ discrimination predicting $\mathrm{T} 2$ gender identity; $\mathrm{T} 1$ internalizing symptoms predicting $\mathrm{T} 2$ discrimination; $\mathrm{T} 1$ internalizing symptoms predicting $\mathrm{T} 2$ gender identity; $\mathrm{T} 1$ gender identity predicting $\mathrm{T} 2$ discrimination; and $\mathrm{T} 1$ gender identity predicting $\mathrm{T} 2$ internalizing symptoms. In all analyses, controls were included for mothers' formal education, adolescents' ethnic minority status and the effects of COVID-19 on school format and family income. Global model fit was assessed using the chi square test of model fit, root mean square error of approximation (RMSEA), comparative fit index (CFI), and the standardized root mean square residual (SRMR).

Finally, to test our third hypothesis that internalizing symptoms might explain (i.e., mediate) the association between discrimination and gender identity, the longitudinal, indirect effects of gender discrimination on gender identity via internalizing distress were tested using the product of coefficients method (multiplication of the $a$ - and $b$-paths; MacKinnon et al. 2002). Building on the path model estimated in the prior analysis, a contemporaneous path from $\mathrm{T} 2$ internalizing symptoms to $\mathrm{T} 2$ gender identity was added. This produced a model in which $\mathrm{T} 1$ gender discrimination predicted internalizing symptoms at $\mathrm{T} 2$ (a longitudinal $a$-path), which in turn predicted gender identity at T2 (contemporaneous $b$-path), while accounting for the effects of internalizing symptoms and gender identity at $\mathrm{T} 1$. Thus, the indirect path $(a b)$ was from $\mathrm{T} 1$ gender discrimination to $\mathrm{T} 2$ gender identity via $\mathrm{T} 2$ internalizing symptoms (see Fig. 1 for specification of the mediation model). The direct path that remains after accounting for $a b$, referred to as $c^{\prime}$, was then used to determine the relative

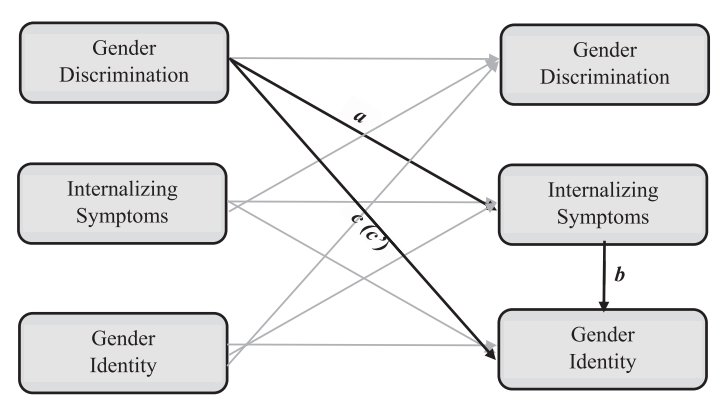

Fig. 1 Conceptual model displaying the longitudinal and bidirectional associations between key study variables. Note. The indirect effect is the product of coefficients, $a b$. The direct effect that remains after accounting for $a b$ is $c$ ', or " $c$-prime". Together, these make up the total effect, $c$, where $c=a b+c$, contribution of the moderated effect (e.g., full or partial mediation). Bias-corrected bootstrapping with 1000 resamples was used to estimated $95 \%$ confidence intervals for direct and indirect effects.

Various methods exist for testing mediation with twooccasion data (Cole \& Maxwell, 2003; see also Rogers et al., 2017 for a relevant example), but this approach was chosen because other methods are either computationally heavy for limited sample sizes, and/or do not allow the estimation of a $c$ ' path (i.e., the direct path between discrimination and gender identity adjusted for the indirect effect), which would prohibit the assessment of the relative degree to which the direct link between discrimination and gender identity was accounted for by internalizing distress (i.e., full or partial mediation). The trade-off of this approach is that the $b$-path is not a longitudinal one, and so conclusive inferences regarding the directionality of the $b$-path cannot be made. However, because the direction of the $b$-path was carefully grounded in theory (PVEST; Spencer et al., 1997), and because this mediation model still included rigorous controls for prior levels of the constructs themselves (autoregressive paths), this overall approach was considered the most optimal under the circumstances.

\section{Results}

\section{Data Screening}

Prior to analyses, data were screened for normality and multivariate outliers. All dependent variables were normally distributed, showing skewness and kurtosis values within $+/-1.00$. Across all variables, there were very few outliers (values falling beyond $+/-3.29$ standard deviations from their respective means), and only one case with outlying values on more than one variable. Missing data were also minimal, with less than $1 \%$ of cases missing values on any single variable, and only three cases missing values on more than one variable $(1.8 \%)$. As such, all cases were retained for analyses and Full Information Maximum Likelihood (FIML) was used to handle missing data (Enders, 2010). Analyses were conducted in Mplus version 8.0 (Muthén \& Muthén, 1998).

\section{Descriptive statistics}

Table 1 displays the means, standard deviations, and zeroorder correlations among all continuous study variables at Time 1. Gender discrimination was common, with $76 \%$ of girls reporting at least one instance. Experiencing gender discrimination was significantly correlated with greater internalizing distress. It was also significantly associated with all three indices of gender identity, and specifically with lower levels of gender identity centrality and perceived 
Table 1 Correlations, means, and standard deviations among continuous variables

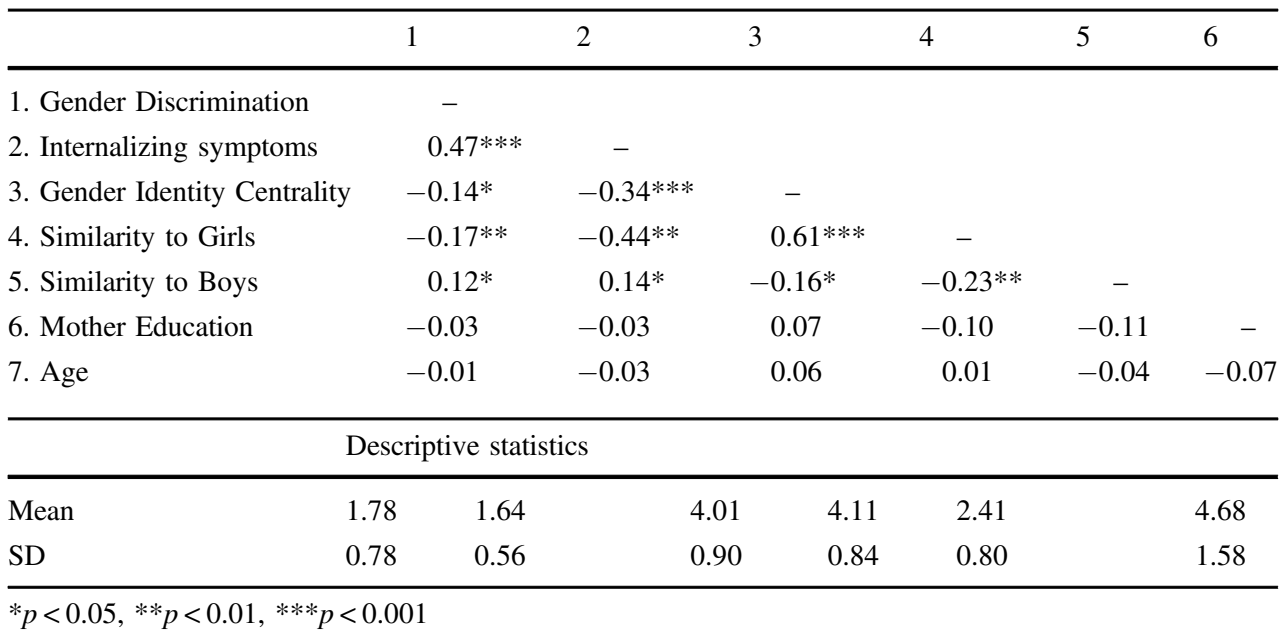

Table 2 Standardized results of cross-lagged panel models specifying relations between gender discrimination, internalizing symptoms, and indicators of gender identity

\begin{tabular}{|c|c|c|c|c|c|c|c|c|c|}
\hline & \multicolumn{3}{|c|}{ Gender centrality } & \multicolumn{3}{|c|}{ Similarity to girls } & \multicolumn{3}{|c|}{ Similarity to boys } \\
\hline & $\beta$ & $95 \% \mathrm{CI}$ & $p$ & $\beta$ & $95 \% \mathrm{CI}$ & $p$ & $\beta$ & $95 \% \mathrm{CI}$ & $p$ \\
\hline \multicolumn{10}{|l|}{ MODEL 1 - CLPM } \\
\hline \multicolumn{10}{|l|}{ Autoregressive Paths } \\
\hline $\mathrm{T} 1$ Disc $\rightarrow$ T2 Disc & 0.51 & {$[0.38,0.63]$} & $<0.001$ & 0.51 & {$[0.38,0.63]$} & $<0.001$ & 0.51 & {$[0.39,0.64]$} & $<0.001$ \\
\hline $\mathrm{T} 1 \mathrm{Int} \rightarrow \mathrm{T} 2 \mathrm{Int}$ & 0.64 & {$[0.52,0.75]$} & $<0.001$ & 0.62 & {$[0.50,0.74]$} & $<0.001$ & 0.64 & {$[0.53,0.74]$} & $<0.001$ \\
\hline TI GenId $\rightarrow$ T2 GenId & d $\quad 0.42$ & {$[0.29,0.56]$} & $<0.001$ & 0.54 & {$[0.42,0.65]$} & $<0.001$ & 0.51 & {$[0.39,0.63]$} & $<0.001$ \\
\hline \multicolumn{10}{|l|}{ Cross-lagged Paths } \\
\hline $\mathrm{T} 1 \mathrm{Disc} \rightarrow \mathrm{T} 2$ Int & 0.15 & {$[0.05,0.26]$} & 0.016 & 0.15 & {$[0.05,0.26]$} & 0.016 & 0.16 & {$[0.05,0.26]$} & 0.013 \\
\hline T1 Disc $\rightarrow$ T2 GenId & -0.05 & {$[-0.20,0.10]$} & 0.515 & -0.15 & {$[-0.28,-0.03]$} & 0.021 & 0.08 & {$[-0.08,0.23]$} & 0.325 \\
\hline $\mathrm{T} 1 \mathrm{Int} \rightarrow \mathrm{T} 2 \mathrm{Disc}$ & 0.20 & {$[0.05,0.35]$} & 0.008 & 0.18 & {$[0.05,0.33]$} & 0.022 & 0.17 & {$[0.05,0.28]$} & 0.020 \\
\hline $\mathrm{T} 1 \mathrm{Int} \rightarrow \mathrm{T} 2$ GenId & -0.10 & {$[-0.26,0.07]$} & 0.236 & -0.15 & {$[-0.27,-0.03]$} & 0.039 & 0.01 & {$[-0.14,0.16]$} & 0.894 \\
\hline T1 GenId $\rightarrow$ T2 Disc. & . 0.10 & {$[-0.03,0.23]$} & 0.141 & 0.04 & {$[-0.10,0.18]$} & 0.584 & -0.06 & {$[-0.18,0.07]$} & 0.376 \\
\hline T1 GenId $\rightarrow$ T2 Int. & 0.01 & {$[-0.11,0.12]$} & 0.937 & -0.03 & {$[-0.15,0.10]$} & 0.680 & -0.05 & {$[-0.17,0.06]$} & 0.339 \\
\hline Model Fit & \multicolumn{9}{|c|}{$\begin{array}{l}X^{2}(7)=5.136, p=0.53 ; R M S E A=\quad X^{2}(7)=6.07, p=0.41 ; R M S E A=0.01 \quad X^{2}(7)= \\
0.01[0.00,0.09] ; C F I=0.99 ; \text { SRMR }=[0.00,0.10] ; C F I=0.99 ; S R M R=0.04 \quad[0.00, \\
0.04\end{array}$} \\
\hline
\end{tabular}

Note. Significant $p$-values are bolded for convenience. GenID = gender identity construct of specific model (e.g., similarity to same gender peers in the first model); Disc = Gender discrimination; Int. = Internalizing symptoms. For parsimony, only key paths are presented (e.g., exogenous covariances and controls are omitted from this display)

similarity to girls, and higher levels of perceived similarity to boys.

\section{Longitudinal associations between gender discrimination, internalizing symptoms, and gender identity}

The first hypothesis was that gender discrimination would positively predict later internalizing symptoms. Results of the auto-regressive cross-lagged path models are displayed in Table 2 (standardized estimates, 95\% confidence intervals, $p$-values, and fit indices). In all three models, there were unique, reciprocal relations between gender discrimination and internalizing symptoms. Girls who reported more discrimination at Time 1 reported greater internalizing symptoms one year later, controlling for their baseline levels of internalizing distress. Similarly, girls who reported more internalizing distress at Time 1 also reported greater incidence of gender discrimination one year later, adjusted for baseline levels of discrimination. None of the controls were significant predictors in any of the models. Parent education, ethnic minority status, age, school format, and reduced family income due to COVID-19 were not significant predictors of gender discrimination, internalizing problems, or gender identity indicators.

The second hypothesis was that gender discrimination would be longitudinally associated with gender identity, 
though directionality was exploratory. There were some significant, longitudinal associations with gender identity, but patterns depended on the construct of interest. In the model for similarity to girls, there was a significant and negative association with discrimination. Girls reporting greater discrimination at $\mathrm{T} 1$, and girls reporting greater internalizing distress at $\mathrm{T} 1$, perceived less similarity to other girls one year later. The reverse directions were not significant: perceived similarity to other girls did not predict later discrimination or later internalizing challenges. There were no other significant, longitudinal associations between gender identity indicators (gender centrality, similarity to boys) and discrimination or internalizing symptoms.

As a sensitivity analysis, the models were rerun with all three indicators of mental health separately, as opposed to using a composite for internalizing symptoms. This resulted in nine total models (three indicators of mental health by three indicators of gender identity), which ultimately showed very minor statistical differences compared to the models previously reported, and clearly evidenced the same overall patterns. Therefore, for parsimony, the prior three models that used the composite variable for internalizing symptoms were considered as the final models.

\section{Mediation by internalizing symptoms}

The third hypothesis was that the associations between gender discrimination and gender identity would be indirect and mediated by internalizing symptoms. In the model predicting gender identity centrality, the indirect effect through internalizing symptoms was negative and statistically significant, $a b=-0.05,95 \% \mathrm{CI}[-0.12,-0.02]$. The remaining direct effect was not significant, $c^{\prime}=-0.01,95 \%$ CI $[-0.16,0.13]$, indicating that the negative association between gender discrimination and gender identity centrality was fully mediated by greater internalizing symptoms. Girls who experienced more frequent gender discrimination at Time 1 reported lower gender identity centrality at Time 2 , which was explained by their greater symptoms of depression, anxiety, and loneliness.

In the model predicting perceived similarity to girls, the indirect effect between gender discrimination and similarity to girls was not significant, $a b=-0.03,95 \%$ CI $[-0.10,0.00]$. Thus, the negative association between gender discrimination and similarity to other girls was not mediated, but rather a direct one. Finally, the indirect effect for perceived similarity to boys was significant and positive, $a b=0.04,95 \%$ CI $[0.01,0.12]$. The direct effect was not significant, $c^{\prime}=0.02,95 \%$ CI $[-0.12$, 0.21 , indicating that the association between gender discrimination and perceived similarity to boys was fully explained by internalizing symptoms.

\section{Discussion}

Gender-based discrimination is a common feature of girls' developmental years (Leaper \& Brown, 2018), and it is thus a unique and salient stressor in girls' lives. This may be particularly relevant during adolescence, which ushers in cognitive advancements that make discrimination more readily perceivable (Brown \& Bigler, 2005). However, few studies have examined how experiences of gender discrimination during this period shape the development of girls' mental health and gender identity, despite adolescence being perhaps one of the most important times for developing a social identity and for achieving emotional resiliencies. Guided by PVEST (Spencer et al., 1997), this study examined the longitudinal and bidirectional associations between adolescent girls' experiences of discrimination and their internalizing symptoms (e.g., anxiety and depressive symptoms, loneliness), as well as indices of their gender identity (e.g., gender centrality, perceived similarity to girls, perceived similarity to boys). Furthermore, because PVEST suggests that changes in identity may be in response to the psychological distress engendered by discrimination, the study examined whether internalizing symptoms mediated these effects. The results showed significant longitudinal associations between elevated levels of gender discrimination and greater internalizing symptoms. They also suggested that higher gender discrimination predicted longitudinal declines in identification with one's own gender groupy, an effect that was typically explained indirectly through internalizing symptoms.

\section{Bidirectional Associations Between Gender Discrimination and Internalizing Symptoms}

Consistent with hypotheses, there was a positive relation between gender discrimination at Time 1 and internalizing symptoms at Time 2. Girls who had more frequent experiences with gender discrimination at the first assessment reported more depressive symptoms, anxiety symptoms, and loneliness one year later, independent of their prior mental health status. This effect is consistent with prior research that documents robust links between discrimination and emotional well-being in racial/ethnic minority youth (Benner et al., 2018) as well as adult women (Hackett et al., 2019), verifying this as a reality for adolescent girls as well. Gender discrimination may undermine girls' locus of control and their beliefs about justice and fairness. Further, discrimination can cause the attenuation of social ties and social capital (Andrews et al., 2018). Altogether, these signal a greater risk for mental health difficulties.

This finding was not particularly surprising, but it is noteworthy for its implications on the developing 
adolescent. Adolescence is a period in which mental health challenges are more common (Merikengas et al., 2010). The prevalent incidence of discrimination during this time (Leaper \& Brown, 2008) may only serve to exacerbate these trends. This is particularly relevant in the light of recent data, which suggests that systemic sexism can statistically account for the greater incidence of mental health problems in women compared to men (Harnois \& Bastos, 2018). Although one cannot draw definitive conclusions from the present data, the findings may evince a similar phenomenon among adolescent girls, who experience higher rates of depression and anxiety than boys (Merikangas et al., 2010). Future work is needed to understand how gender discrimination may contribute to gender disparities in adolescents' psychological and emotional health.

Interestingly, the results also showed that internalizing symptoms at Time 1 predicted greater gender discrimination at Time 2. Girls higher in depressive and anxiety symptoms reported elevated levels of gender discrimination one year later. This finding was less anticipated but indicates a bidirectional link that has not been tested in prior work. At least two explanations seem plausible. First, it is possible that individuals experiencing internalizing distress may experience social interactions in a more negative light. A person's affective states might shape perceptions of their social interactions and relationships, and so individuals experiencing greater difficulties around depression and anxiety, for example, might be more perceiving or more aware of discriminatory intentions in others. However, this is likely an incomplete explanation because the path in the opposite direction was also significant (described previously); altogether, the findings likely indicate more of an ongoing, reciprocating process.

This latter explanation would dovetail with themes readily observed across the developmental sciences regarding feedback loops in peer interactions that heighten negative treatment from others. For example, bullied and harassed youth are more likely to suffer emotionally and psychologically, and these emotional and psychological struggles render them a further target for continuing negative treatment (Da Silva et al., 2020). It is possible that a similar process is at play when girls experience discrimination. When faced with discriminatory events, girls experience internalizing distress, which may make them more vulnerable to continued negative treatment. Research shows that when girls experience discrimination, they may often engage avoidant responses, such as dismissing the event or internalizing the sexism, out of fear of future discrimination. These avoidant responses may leave open the door for continued sexist treatment in the long term (Kaiser \& Miller, 2004). Ultimately, more work is needed to unpack the mechanisms behind these bidirectional associations, but the findings underscore the value in considering the relations between discrimination and mental health as being cyclical in nature.

\section{Gender Discrimination, Distress, and Gender Identity Development}

The second major hypothesis, in line with PVEST, was that gender discrimination might lead to changes in gender identity. Although no directional predictions were made regarding girls' gender identity, the findings were relatively clear: most girls in the sample identified less with their gender group one year following their experiences with discrimination. Specifically, girls who reported more frequent experiences with discrimination at Time 1 reported one year later that their gender was a less salient part of their overall identity, that they felt less similar to other girls, and that they felt more similar to boys. Importantly, the effect in the other direction was not evident in the data, as no indices of gender identity at Time 1 were associated with later experiences with discrimination at Time 2 .

At face value, these patterns seem intuitive. Gender discrimination targets girls for their gender group, and so many girls may perceive their femininity as a liability for future discrimination. However, these systematic changes in identity only really make sense in the context of the systems of oppression that adolescent girls regularly occupy. From a PVEST (Spencer et al., 1997) standpoint, girls' dissociation from their gender identity is in response to gendered power arrangements in their social ecologies. Gender discrimination does not happen in a vacuum, but within systems that devalue feminine-typed traits and qualities while elevating masculine-typed ones. As such, when girls are confronted with sexism, their efforts to cope and socially position themselves (e.g., gender expression and identity) are responsive to prevailing sexist norms and standards.

Importantly, in two of the models (gender identity centrality, similarity to boys), the longitudinal associations between discrimination and gender identity were indirect, being completely mediated by girls' greater internalizing symptoms. In this sense, the findings support PVEST in its assertions that changes in social identity following discrimination represent a coping response, intended to assuage the psychological distress engendered by discrimination. When the girls in the study experienced discrimination to the point that it increased feelings of depression, anxiety, and loneliness, they perceived their gender as less central to their overall identity and felt more similar to boys.

These findings pose critical implications for research and practice because gender identity can be a source of social capital and psychological protection for girls (Spencer et al., 1997). A greater sense of belonging with other girls and women is an important ingredient of a feminist identity and 
can provide empowerment and psychological resources for coping with sexism (Leaper \& Arias, 2011). However, the findings suggest that gender discrimination may erode these resources over time. Furthermore, dissociating from one's gender collective likely will not reduce future discrimination, as evidenced by the findings that gender identity did not predict later discrimination. Thus, gender discrimination will likely happen regardless of a girl's gender expression, and de-identifying with their gender collective may only serve to further isolate girls from supportive networks and undermine their sense of solidarity with other girls and women. Implied is the need for preventions and interventions that teach girls how to effectively cope with gender discrimination, while also reducing actual prevalence and exposure.

\section{Limitations}

The study benefited from a longitudinal design that facilitated the estimation of prospective and bidirectional associations and allowing the more rigorous control of prior time points. However, the two-occasion data introduced some constraints in estimating mediated effects, specifically the estimation of a concurrent $b$-path as opposed to a longitudinal one. This meant that the model could not verify the directionality of the $b$-path. The proposed directionality was consistent with theory (Spencer et al., 1997) and extant empirical work (Hewlett et al., 2008), but future studies should verify these directions with a third time point.

The relatively small sample size restricted an ability to examine the role of other important social identities-particularly race and ethnicity-in shaping the processes of interest. From an intersectionality perspective (Crenshaw, 1991), girls' experiences with gender discrimination are uniquely shaped by social positions salient to their other identities, including race/ ethnicity. Gendered norms and standards in the United States often take whiteness for granted (Ghavami and Peplau 2013), and so girls' experiences with discrimination contain inherently racialized messaging. Furthermore, adolescent girls of color experience sexism and sexual harassment at rates higher than white girls (Goldstein et al., 2007). The result is that experiences with gender discrimination may affect girls of color in unique ways. Although the models accounted for the effect of racial/ethnic minority status as a control and found no differences in the prediction of all study constructs, intentional efforts must be taken to assess the unique experiences of adolescent girls of color in more depth.

Finally, the study design did not allow for the consideration of features of adolescents' social systems (e.g., school environments) in which the discrimination is likely happening. Systems, like individuals, vary in the level of sexism that they perpetuate. It is possible that in geographic regions or in schools where sexism is more structurally engrained, these associations at the individual level may be more or less pronounced. Future studies may consider how girls' individual and subjective experiences are shaped by broader features of their social contexts.

\section{Conclusion}

For most girls, experiencing gender discrimination is an inevitable reality of their adolescent years. However, there is a notable lack of research about how gender discrimination can shape girls' emotional well-being and their sense of identity, which are key features of this developmental period. Therefore, this study examined the longitudinal and bidirectional associations between girls' experiences of gender discrimination and indices of their emotional well-being and gender identity. The results showed that girls who reported greater discrimination suffered more from internalizing problems one year later. This process, in turn, often explained girls' tendency to deidentify with their own gender group, likely as an attempt to cope with sexism and reduce its future occurrence. This study underscores the importance of continued efforts to address sexism and gender discrimination in adolescence.

Authors' contributions AR conceived of the study, collected the data, performed the statistical analyses and interpretation of results, and led the writing of the manuscript; RC conceived of the study and assisted in the interpretation of results and the writing of the manuscript; KG assisted in writing and editing the manuscript. All authors read and approved the final manuscript.

Data Sharing and Declaration This manuscript's data will not be deposited. The authors are willing to share their data upon reasonable request.

\section{Compliance with Ethical Standards}

Conflict of Interest The authors declare no competing interests.

Ethical Approval All procedures involving human participants were performed in accordance with the ethical standards of the institution and/or national research committee and with the 1964 Helsinki declaration and its later amendments or comparable ethnical standards.

Informed Consent All adolescents in the study assented to participation; consent was obtained from each participants' primary caregiver.

Publisher's note Springer Nature remains neutral with regard to jurisdictional claims in published maps and institutional affiliations.

\section{References}

Allgaier, A. K., Frühe, B., Pietsch, K., Saravo, B., Baethmann, M., \& Schulte-Körne, G. (2012). Is the children's depression inventory short version a valid screening tool in pediatric care? A 
comparison to its full-length version. Journal of Psychosomatic Research, 73(5), 369-374. https://doi.org/10.1016/j.jpsychores. 2012.08.016.

Andersson, M. A., \& Harnois, C. E. (2020). Higher exposure, lower vulnerability? The curious case of education, gender discrimination, and women's health. Social Science \& Medicine, 246, 112780. https://doi.org/10.1016/j.socscimed.2019.112780.

Andrews, N. C., Santos, C. E., Cook, R. E., \& Martin, C. L. (2018). Gender discrimination hinders other-gender friendship formation in diverse youth. Journal of Applied Developmental Psychology, 59(5), 16-25. https://doi.org/10.1016/j.appdev.2018.02.006.

Ayres, M. M., Friedman, C. K., \& Leaper, C. (2009). Individual and situational factors related to young women's likelihood of confronting sexism in their everyday lives. Sex Roles, 61(7-8), 449-460. https://doi.org/10.1007/s11199-009-9635-3.

Baysu, G., Phalet, K., \& Brown, R. (2014). Relative group size and minority school success: The role of intergroup friendship and discrimination experiences. British Journal of Social Psychology, 53(2), 328-349. https://doi.org/10.1111/bjso.12035.

Bell, A. N., \& Juvonen, J. (2020). Gender discrimination, perceived school unfairness, depressive symptoms, and sleep duration among middle school girls. Child Development, 91(6), 1865-1876. https://doi.org/10.1111/cdev.13388.

Benner, A. (2017). The toll of racial/ethnic discrimination on adolescents' adjustment. Child Development Perspectives, 11(4), 251-256. https://doi.org/10.1111/cdep.12241.

Benner, A. D., Wang, Y., Shen, Y., Boyle, A. E., Polk, R., \& Cheng, Y. P. (2018). Racial/ethnic discrimination and well-being during adolescence: A meta-analytic review. American Psychologist, 73 (7), 855-883. https://doi.org/10.1037/amp0000204.supp.

Bronfenbrenner, U., \& Morris, P.A. (2006). The bioecological model of human development. In W. Damon (Series Ed.) \& R.M. Lerner (Vol. Ed.), Handbook of child psychology: Theoretical models of human development (pp. 793-828). New York, NY: Wiley.

Brown, C. S., \& Bigler, R. S. (2005). Children's perceptions of discrimination: A developmental model. Child Development, 76(3), 533-553. https://doi.org/10.1111/j.1467-8624.2005.00862.x.

Cassidy, C., O'Connor, R. C., Howe, C., \& Warden, D. (2004). Perceived discrimination and psychological distress: The role of personal and ethnic self-esteem. Journal of Counseling Psychology, 51(3), 329-339. https://doi.org/10.1037/0022-0167.51. 3.329 .

Cole, D. A., \& Maxwell, S. E. (2003). Testing mediational models with longitudinal data: questions and tips in the use of structural equation modeling. Journal of Abnormal Psychology, 112(4), 558-577. https://doi.org/10.1037/0021-843X.112.4.558.

Crenshaw, K. (1991). Mapping the margins: Identity politics, intersectionality, and violence against women. Stanford Law Review, 43(6), 1241-1299. https://doi.org/10.2307/1229039.

Da Silva, M. A., Gonzalez, J. C., Person, G. L., \& Martins, S. S. (2020). Bidirectional association between bullying perpetration and internalizing problems among youth. Journal of Adolescent Health, 66(3), 315-322. https://doi.org/10.1016/j.jadohealth. 2019.09.022

DeLay, D., Martin, C. L., Cook, R. E., \& Hanish, L. D. (2018). The influence of peers during adolescence: Does homophobic name calling by peers change gender identity? Journal of Youth and Adolescence, 47(3), 636-649. https://doi.org/10.1007/s10964017-0749-6.

DeNavas-Walt, C., Proctor, B.D., \& Smith, J.C. (2013). Income, Poverty, and Health Insurance Coverage in the United States: 2012. Current Population Reports P60-245. US Census Bureau. https://doi.org/10.1037/e561322006-001.

Enders, C. K. (2010). Applied missing data analysis. New York, NY: Guilford Press.
Ghavami, N., \& Peplau, L. A. (2013). An intersectional analysis of gender and ethnic stereotypes: Testing three hypotheses. Psychology of Women Quarterly, 37(1), 113-127. https://doi.org/10. 1177/0361684312464203.

Goldstein, S. E., Malanchuk, O., Davis-Kean, P. E., \& Eccles, J. S. (2007). Risk factors of sexual harassment by peers: A longitudinal investigation of African American and European American adolescents. Journal of Research on Adolescence, 17(2), 285-300. https://doi.org/10.1111/j.1532-7795.2007.00523.x.

Grollman, E. A. (2012). Multiple forms of perceived discrimination and health among adolescents and young adults. Journal of Health and Social Behavior, 53(2), 199-214. https://doi.org/10. $1177 / 0022146512444289$.

Hackett, R. A., Steptoe, A., \& Jackson, S. E. (2019). Sex discrimination and mental health in women: A prospective analysis. Health Psychology, 38(11), 1014 https://doi.org/10.1037/hea 0000796.

Harnois, C. E., \& Bastos, J. L. (2018). Discrimination, harassment, and gendered health inequalities: do perceptions of workplace mistreatment contribute to the gender gap in self-reported health? Journal of Health and Social Behavior, 59(2), 283-299. https:// doi.org/10.1177/0022146518767407.

Hewlett, S. A., Luce, C. B., Servon, L. J., Sherbin, L., Shiller, P., Sosnovich, E., \& Sumberg, K. (2008). The Athena factor: Reversing the brain drain in science, engineering, and technology. Harvard Business Review Research Report, 10094, 1-100.

Hughes, M. E., Waite, L. J., Hawkley, L. C., \& Cacioppo, J. T. (2004). A short scale for measuring loneliness in large surveys: Results from two population-based studies. Research on aging, 26(6), 655-672. https://doi.org/10.1177/0164027504268574.

Kaiser, C. R., \& Miller, C. T. (2004). A stress and coping perspective on confronting sexism. Psychology of Women Quarterly, 28(2), 168-178. https://doi.org/10.1111/j.1471-6402.2004.00133.x.

Killen, M., Lee-Kim, J., McGlothlin, H., Stangor, C., \& Helwig, C. C. (2002). How children and adolescents evaluate gender and racial exclusion. Monographs of the society for Research in Child Development, 67(4), 1-129.

Kim, E., \& Park, H. (2018). Perceived gender discrimination, belief in a just world, self-esteem, and depression in Korean working women: A moderated mediation model. Women's Studies International Forum, 69, 143-150. https://doi.org/10.1016/j.wsif. 2018.06.006.

Klonoff, E., \& Landrine, H. (1995). The schedule of sexist events: A measure of lifetime and recent sexist discrimination in women's lives. Psychology of Women Quarterly, 19(4), 439-472. https:// doi.org/10.1111/j.1471-6402.1995.tb00086.x.

Leaper, C., \& Brown, C. S. (2008). Perceived experiences with sexism among adolescent girls. Child Development, 79(3), 685-704. https://doi.org/10.1111/j.1467-8624.2008.01151.x.

Leaper, C., \& Arias, D. M. (2011). College women's feminist identity: A multidimensional analysis with implications for coping with sexism. Sex Roles, 64(7-8), 475-490. https://doi.org/10.1007/ s11199-011-9936-1.

Leaper, C., \& Brown, C. S. (2018). Sexism in childhood and adolescence: Recent trends and advances in research. Child Development Perspectives, 12(1), 10-15. https://doi.org/10.1111/cdep. 12247.

Leaper, C., Farkas, T., \& Brown, C. S. (2012). Adolescent girls' experiences and gender-related beliefs in relation to their motivation in math/science and English. Journal of Youth and Adolescence, 41(3), 268-282. https://doi.org/10.1007/s10964-0119693-z.

MacKinnon, D. P., Lockwood, C. M., Hoffman, J. M., West, S. G., \& Sheets, V. (2002). A comparison of methods to test mediation and other intervening variable effects. Psychological Methods, 7(1), 83-104. https://doi.org/10.1037/1082-989X.7.1.83. 
Martin, C. L., Andrews, N. C., England, D. E., Zosuls, K., \& Ruble, D. N. (2017). A dual identity approach for conceptualizing and measuring children's gender identity. Child Development, 88(1), 167-182. https://doi.org/10.1111/cdev.12568.

McKown, C., \& Weinstein, R. S. (2003). The development and consequences of stereotype consciousness in middle childhood. Child Development, 74(2), 498-515. https://doi.org/10.1111/ 1467-8624.7402012.

Merikangas, K. R., He, J. P., Burstein, M., Swanson, S. A., Avenevoli, S., Cui, L., \& Swendsen, J. (2010). Lifetime prevalence of mental disorders in US adolescents: results from the national comorbidity survey replication-adolescent supplement (NCS-A). Journal of the American Academy of Child \& Adolescent Psychiatry, 49(10), 980-989. https://doi.org/10.1016/j.jaac.2010.05.017.

Muthén, L. K., \& Muthén, B. O. (1998). Mplus user's guide. Seventh edition (pp. 2018. Los Angeles: Muthén \& Muthén.

Perry, D. G., Pauletti, R. E., \& Cooper, P. J. (2019). Gender identity in childhood: A review of the literature. International Journal of Behavioral Development, 43(4), 289-304. https://doi.org/10. $1177 / 0165025418811129$.

Robnett, R. D. (2016). Gender bias in STEM fields: Variation in prevalence and links to STEM self-concept. Psychology of Women Quarterly, 40(1), 65-79. https://doi.org/10.1177/ 0361684315596162.

Rogers, A. A., DeLay, D., \& Martin, C. L. (2017). Traditional masculinity during the middle school transition: Associations with depressive symptoms and academic engagement. Journal of youth and adolescence, 46(4), 709-724.

Rogers, A. A., Nielson, M. G., \& Santos, C. E. (2021c). Manning up while growing up: A developmental-contextual perspective on masculine gender-role socialization in adolescence. Psychology of Men \& Masculinities, 22(2), 354 https://doi.org/10.1037/ men0000296.

Rogers, A. A., Ha, T., \& Ockey, S. (2021b). Adolescents' perceived socio-emotional impact of COVID-19 and implications for mental health: results from a US-based mixed-methods study. Journal of Adolescent Health, 68(1), 43-52. https://doi.org/10. 1016/j.jadohealth.2020.09.039.

Rogers, A. A., Boyack, M., Cook, R. E., \& Allen, E. (2021a). School Connectedness and STEM orientation in sdolescent girls: The role of perceived gender discrimination and implicit genderscience stereotypes. Sex Roles, 85, 405-421. https://doi.org/10. 1007/s11199-021-01224-7.

Santos, C. E., \& Toomey, R. B. (2018). Integrating an intersectionality lens in theory and research in developmental science. New
Directions for Child and Adolescent Development, 161, 7-15. https://doi.org/10.1002/cad.20245.

Scottham, K. M., Sellers, R. M., \& Nguyên, H. X. (2008). A measure of racial identity in African American adolescents: The development of the multidimensional inventory of black identity-teen. Cultural Diversity and Ethnic Minority Psychology, 14(4), 297 https://doi.org/10.1037/1099-9809.14.4.297.

Spencer, M. B., Dupree, D., \& Hartmann, T. (1997). A phenomenological variant of ecological systems theory (PVEST): A self-organization perspective in context. Development and Psychopathology, 9(4), 817-833. https://doi.org/10.1017/s0954579497001454.

Spitzer, R. L., Kroenke, K., Williams, J. B., \& Löwe, B. (2006). A brief measure for assessing generalized anxiety disorder: the GAD-7. Archives of Internal Medicine, 166(10), 1092-1097. https://doi.org/10.1001/archinte.166.10.1092.

Tiirikainen, K., Haravuori, H., Ranta, K., Kaltiala-Heino, R., \& Marttunen, M. (2019). Psychometric properties of the 7-item generalized anxiety disorder scale (GAD-7) in a large representative sample of Finnish adolescents. Psychiatry Research, 272, 30-35. https://doi.org/10.1016/j.psychres.2018.12.004.

Adam A. Rogers is an Assistant Professor in the School of Family Life at Brigham Young University. His research focuses on the development of competence and psychopathology in adolescence, with an emphasis on gender socialization and development.

Rachel E. Cook Rachel E. Cook is a post-doctoral scholar at the Institute for Child Development and Family Relations at California State University, San Bernardino. She studies the multidimensionality of gender identity and the developmental and contextual influences on the emergence of gender-related prejudices.

Kaitlyn Guerrero is an undergraduate student at Brigham Young University studying Human Development in the School of Family Life. Her research interests include healthy sexuality and gender socialization. 